\title{
Um Estudo de Mapeamento das Contribuições e Desafios de Pesquisa em Máquinas Sociais
}

\section{A Mapping Study of the Contributions and Research Challenges in Social Machines}

\author{
Roosevelt Fabiano Moraes da Silva ${ }^{1}$, Vanilson André de Arruda Burégio ${ }^{2}$ \\ ${ }^{1}$ CESAR School, Centro de Estudos e Sistemas Avançados do Recife, Recife, Brasil \\ ${ }^{2}$ Departamento de Computação, Universidade Federal Rural de Pernambuco, Recife, Brasil \\ Correspondência: Roosevelt Fabiano Moraes da Silva, Centro de Estudos e Sistemas Avançados do Recife, \\ Endereço: Cais do Apolo, 77, Bairro do Recife, CEP.: 50.030-390 Recife, Brasil. Tel: 55 81 3419-6700. E-mail: \\ roosevelt.silva@ifms.edu.br
}

Recebido: 5 de outubro de 2018 Aceito: 30 de novembro de 2018 Publicado: 31 de dezembro de 2018

DOI: http://dx.doi.org/10.21714/1679-18272018v16Ed.p245-257

\begin{abstract}
Resumo
O campo das máquinas sociais ainda é uma área nova, sem uma definição que tenha o senso comum dos pesquisadores, isso cria algumas dificuldades para entender qual o conceito, ou qual projeto de máquinas sociais devemos escolher. Este trabalho tem como objetivo investigar de forma detalhada, os trabalhos apresentados nas oficinas de máquinas sociais e nas principais bases de dados, a partir desta análise criamos um mapa de estudo, um esquema de categorização das obras e também gráficos de bolhas entre as categorizações. Foi produzido panorama da área de máquinas sociais, através da identificação dos principais tópicos, tipos, tendências e desafios através desta pesquisa preliminar, além de definir a maturidade das contribuições existentes. Este trabalho fornece uma base conceitual para a compreensão da área e também detecta os problemas de pesquisa e lacunas existentes no campo das máquinas sociais.
\end{abstract}

Palavras-chave: Máquinas sociais, Caracterização, Mapeamento.

\begin{abstract}
The field of social machines is still a new area, without a definition that has the common sense of researchers, this creates some difficulties to understand which concept, or which social machine project we should choose. This work aims to investigate in detail the work presented in the social machine workshops and in the main databases, from this analysis we create a study map, a categorization scheme of the works and also graphs of bubbles between the categorizations. It was produced a landscape of the area of social machines, was by identifying the main topics, types, trends and challenges through this preliminary research, as well as defining the maturity of existing contributions. This paper provides a conceptual basis for understanding the area and also detects research problems and gaps in the field of social machines.
\end{abstract}

Keywords: Social Machines, Characterizing, Mapping.

Esta obra está licenciada sob uma Licença Creative Commons Attribution 3.0.

\section{Introdução às Máquinas Sociais}

De acordo com BURÉGIO, MEIRA e ROSA (2013), hoje em dia cada vez mais, os meios de computação são utilizados para conectar as pessoas, seja com outras pessoas ou com as máquinas, essa filosofia de conexão mudou a semântica da computação. É interessante salientar que a internet é composta por um conjunto gigantesco de coisas, formada por elementos concretos e abstratos, entre essas coisas se destacam, por exemplo: humanos, máquinas, informações, tecnologias, padrões, regras, governos e outros, sendo que tudo está em constante evolução 
e conversando entre si (TINATI et al., 2013).

Há inúmeras discussões e diversas interpretações de conceptualização de máquinas sociais, porém uma definição simples pode ser descrita da seguinte maneira: é um paradigma que pode lidar com a complexidade da internet nos tempos modernos, com seu crescimento em todos os lugares, e de certa forma, deve ser capaz de conectar e se entender com cada entidade interligada, simplificando esse conceito, uma máquina social é uma entidade que combina processos computacionais e sociais. (BURÉGIO; MEIRA; ROSA, 2013).

As máquinas sociais apareceram de alguma forma na internet, e sua aparição teve uma força maior com o surgimento da Internet 2.0, principalmente através da popularização de blogs pessoais, redes sociais e sites de compartilhamento de mídia, mas o termo máquina social ainda é confuso e desconhecido para muitos (BURÉGIO; MEIRA; ROSA, 2013).

Outra definição de máquina social é visualizada da seguinte forma: ao invés de colocar as partes humanas e as partes computacionais separadas por uma linha fina, um círculo deve ser desenhado, então adiciona as duas partes dentro deste circulo, fazendo com que as partes interajam, comuniquem e se ajudem (SHADBOLT et al., 2013).

De maneira mais técnica há uma outra definição, onde diz que as máquinas sociais são as redes de interações sociais que trabalham com as redes de estruturas de comunicação da internet, isto é, redes sócio técnicas que usam a internet e todos os seus recursos, sejam esses recursos relacionados com dados, velocidade, disponibilidade e acessibilidade, disponibilizados de maneira organizada, abundante e para todos (DALTON, 2013).

Este estudo de mapeamento busca direcionar o cenário nesses últimos anos, afim de trazer informações, desafios e lacunas que esta área pode proporcionar. Portanto, como resultado deste mapeamento, pretende-se ter uma visão geral dos estudos que estão sendo realizados em máquinas sociais, bem como a classificar as contribuições através do foco da pesquisa, tipo de pesquisa e tipo de contribuição, e por fim verificando as lacunas existentes.

As próximas seções estão organizadas da seguinte forma: a Seção 2 discute alguns trabalhos relacionados sobre o tema. A Seção 3 demonstra a metodologia utilizada na construção do mapeamento do estudo. A Seção 4 apresenta os resultados obtidos no estudo de mapeamento em máquinas sociais. A Seção 5 discute vários pontos importantes e relevantes da pesquisa realizada, já a Seção 6 possui as últimas observações e as lacunas encontradas.

\section{Trabalhos Relacionados}

Antes de iniciar o mapeamento sistemático, os esforços foram concentrados em encontrar estudos de mapeamento sistemático que analisasse e caracterizasse as pesquisas com máquinas sociais; após esse esforço foi confirmado que tais estudo ainda não existiam.

Há alguns trabalhos que visam principalmente a compreensão, conceptualização, estudo e caracterização de máquinas sociais; estes são os trabalhos que são relacionados nesta seção.

Neste primeiro trabalho aqui relacionado, BURÉGIO, MEIRA e ROSA, (2013) caracteriza máquinas sociais como o resultado da convergência de três visões: software social, pessoas como unidades computacionais e software como entidades sociáveis.

O software social é um sistema que permite interação e colaboração humana, através de textos e mídias, são sistemas que permitirão uma nova maneira de desenvolver software, usando e reutilizando várias API de terceiros para, assim criando uma nova aplicação. As pessoas como uma unidade computacional, são pessoas que trabalham com suas habilidades e interagem com a máquina para resolver problemas simples do ponto de vista humano, mas complexas do ponto de vista computacional. O software como uma entidade sociável, significa a socialização do software, ou seja, dois ou mais sistemas conversando (BURÉGIO; MEIRA; ROSA, 2013).

Em outro trabalho ainda em 2013, SHADBOLT et al., (2013) relaciona a caracterização do tema a algumas áreas de conhecimento no âmbito da tecnologia da informação, que de alguma forma interagem com as máquinas sociais, mas não são sinônimas de máquinas sociais.

É essencial aos pesquisadores compreender os conceitos das áreas definidas por SHADBOLT et al., (2013), por exemplo, o conceito de sabedoria de multidões e inteligência coletiva, que não podem ser confundidas com máquinas sociais; a sabedoria das multidões refere-se à tomada de decisão baseada em um grupo de pessoas, já a inteligência coletiva tem um conceito mais amplo, dizendo que as pessoas devem fazer as coisas de maneira coletiva e essas coisas devem ser inteligentes. Nas máquinas sociais, a inteligência humana, e a sabedoria devem se unir, ou seja, tornar-se em uma coisa apenas (SHADBOLT et al., 2013). 
Outro conceito é a computação social, que é predominantemente relacionada à colaboração com suporte por computador, principalmente através de grupos e comunidades na internet. Já nas máquinas sociais, essas características são combinadas com parceiros técnicos e combinados com a colaboração social (SHADBOLT et al., 2013).

Outro tema é a computação humana, que ocorre quando as máquinas usam as pessoas para executar tarefas que os computadores não conseguem realizar, porém o termo crowdsourcing é associado ao conceito de contribuição colaborativa ou coletiva, ou seja, um grupo que se reúne para resolver problemas significativos juntos (SHADBOLT et al., 2013). Por fim há o conceito de inovação aberta, que é trata apenas de um paradigma onde as ideias internas ou externas da empresa devem oferecem um produtor melhor e atual ao mercado, logo, o conjunto de todos esses conceitos unidos caracterizam uma máquina social (SHADBOLT et al., 2013).

Outro trabalho relacionado também em 2013, TINATI et al., (2013) definiu um modelo chamado HTP (Heterogeneous Networks, Translation and Phases), este modelo descreve o desenvolvimento do website Wikipédia, que pelo autor é considerado uma máquina social.

Nesse modelo, a letra H (redes heterogêneas) representa "A Web", que é uma rede heterogênea, formada por diferentes atividades na internet, realizadas por atores (humanos e máquinas), que interagem por interesses comuns e geram resultados. A letra $\mathrm{T}$ (tradução) diz respeito à tradução da rede entre os atores, que estão sempre em um processo de crescimento e alinhamento entre eles. Finalmente, a letra $\mathrm{P}$ (fases) significa que uma máquina social é o produto de várias etapas de tradução, envolvendo o arranjo de várias redes disponíveis. O modelo HTP projeta uma outra maneira de enxergar as máquinas sociais (TINATI et al., 2013).

Em 2015, VASS e MUNSON, (2015) sugeriu uma abordagem alternativa, e mais psicológica sobre o assunto, que prioriza uma visão da atividade sócio técnica como uma formação de "estruturas de projetos reflexivos", demonstrando que a reflexividade nos sistemas sociais é dividida em elementos mais fundamentais (Reconhecimento e Responsabilidade).

A reflexividade dos agentes (humanos e máquinas) é um processo cognitivo complicado, que depende da habilidade dos agentes de auto monitorar e coordenar suas atividades uns sobre os outros com os objetivos do projeto. Os mesmos modelos aplicados às máquinas são tradicionalmente utilizados para humanos da mesma maneira (VASS; MUNSON, 2015).

De acordo com VASS e MUNSON, (2015) a reflexividade tem uma parceria mútua com dois outros conceitos: Reconhecimento e Responsabilidade. O reconhecimento diz como as estruturas do projeto preveem, que os participantes precisam estabelecer ou manter suas "posições sociais", e como essa posição é reconhecida e disponibilizada como um recurso para outros participantes. A responsabilidade refere-se à qualidade e velocidade de reação à atividade eferente dos agentes participantes, mas também aos níveis de reconhecimento dados nas respostas aos participantes. Formando, de acordo com o autor os três "Rs" das Máquinas Sociais: Reflexividade, Reconhecimento e Responsabilidade.

\section{Metodologia da Pesquisa}

O objetivo desta seção é estudar e obter uma visão geral da literatura sobre máquinas sociais, identificando os tipos e as quantidades de pesquisas, bem como os tipos de contribuições disponíveis, utilizando a técnica de estudo de mapeamento, que é uma forma específica de revisão sistemática da literatura (BURÉGIO; MEIRA; ALMEIDA, 2010; GBAFFONOU; LAPALME; CHAMPAGNE, 2015; MELDRUM; LICORISH; SAVARIMUTHU, 2017).

Trabalhando neste formato, é possível classificar e entender melhor o assunto em questão, além de que, este mapa pode ser útil para definir áreas de pesquisas ainda não exploradas pelos pesquisadores e para identificar lacunas nos artigos utilizados (BURÉGIO; MEIRA; ALMEIDA, 2010; GBAFFONOU; LAPALME; CHAMPAGNE, 2015; MELDRUM; LICORISH; SAVARIMUTHU, 2017).

Por essas razões foi decidido adotar essa metodologia, assim foram analisados o formato de alguns trabalhos nas diferentes áreas da Engenharia de Software (BURÉGIO; MEIRA; ALMEIDA, 2010; SANTOS; OLIVEIRA; ALMEIDA, 2015; HASSAN et al., 2016; GBAFFONOU; LAPALME; CHAMPAGNE, 2015 MELDRUM; LICORISH; SAVARIMUTHU, 2017) utilizando os estudos acima como base, foi possível classificar e compreender as máquinas sociais, através da metodologia de estudo de mapeamento. 
A. Pergunta de pesquisa

Um passo essencial em qualquer estudo de mapeamento é a definição de uma questão significativa dentro de um assunto específico. É crucial ter um significado desde o início, definindo assim o problema que orientará, de maneira apropriada, organizada e ordeira, toda a revisão. Então definiu-se à questão central do estudo que é:

"Em que aspectos na área de Máquinas Sociais os pesquisadores focaram estruturar as suas pesquisas, e quais os principais desafios e a maturidade das pesquisas existentes? “

Como pode-se observar no problema de pesquisa da revisão, há duas direções, em uma primeira direção são classificadas as pesquisas dos artigos selecionados, adotando um esquema que seja possível entender as principais abordagens, e os principais assuntos sobre as máquinas sociais nos últimos anos. Na segunda direção, são respondidos quais são os conhecimentos ou habilidades que foram compartilhadas até agora, identificando os principais desafios e lacunas.

B. Seleção de pesquisa

Nesta revisão sistemática, ao invés de selecionar artigos somente através de bases de busca, e então realizar uma inclusão e exclusão de artigos, o que poderia devolver trabalhos mais focadas em uma área ou excluir pesquisas importantes, foram selecionados primeiramente 35 artigos dos workshops sobre teoria e prática de máquinas sociais; esses workshops aconteceram no evento da WWW (World Wide Web) nos anos de 2013 à 2017. Em 2017, o workshop de máquinas sociais tornou-se parte integrante do evento WWW.

Também houve uma seleção de forma tradicional, como diz a cartilha de uma revisão sistemática, 19 artigos das bases ACM (Association for Computing Machinery) e IEEE (Institute of Electrical and Electronic Engineers) entre 2013 e 2017 foram selecionados, adicionando apenas um filtro de busca, as palavras "máquinas sociais" ou "máquina social" contidas somente nos títulos ou no resumo das obras, também sem que houvessem exclusões.

Então ao todo, este estudo trabalha com 54 artigos selecionados, representando uma média de 11 trabalhos por ano, a Tabela 1 apresenta a distribuição de todos os artigos utilizados.

Tabela 1. Distribuição de todos os artigos utilizados por ano

\begin{tabular}{|l|l|l|l|l|l|l|}
\hline & $\mathbf{2 0 1 3}$ & $\mathbf{2 0 1 4}$ & $\mathbf{2 0 1 5}$ & $\mathbf{2 0 1 6}$ & $\mathbf{2 0 1 7}$ & Total \\
\hline Quantidade de trabalhos selecionados & 12 & 12 & 12 & 11 & 7 & 54 \\
\hline
\end{tabular}

O estudo de mapeamento desses trabalhos, deve ser suficiente para que seja possível responder à questão de pesquisa adequadamente.

\section{O Estudo de Mapeamento}

Agora, será elucidado o esquema de classificação construído e utilizado com base nos trabalhos selecionados, além de demonstrar o mapa do estudo e seus passos.

\section{A. Esquema de Classificação}

Foram utilizados os mesmos conceitos de facetas abordadas nas obras (BURÉGIO; MEIRA; ALMEIDA, 2010; SANTOS; OLIVEIRA; ALMEIDA, 2015). O esquema da revisão deste capítulo também possui um esquema de classificação que aborda três facetas: $i$ ) foco da pesquisa; ii) tipo de pesquisa e iii) tipo de contribuição.

A primeira faceta foi inteiramente desenvolvida com base na leitura, identificação e classificação definida pelo autor desta revisão, a fim de descobrir os focos dos trabalhos, a segunda faceta foi totalmente baseada na classificação dos estudos descritos por (BURÉGIO; MEIRA; ALMEIDA, 2010; SANTOS; OLIVEIRA; ALMEIDA, 2015), segundo eles, este aspecto investiga a forma como o trabalho foi realizado, podendo também ser reutilizado em qualquer estudo de mapeamento e classificação, e finalmente na terceira faceta, foi realizado um uso da tabela de classificação de (BURÉGIO; MEIRA; ALMEIDA, 2010; SANTOS; OLIVEIRA; ALMEIDA, 2015), também com novas definições com base nos trabalhos da área de máquinas sociais, esta faceta visa identificar o resultado das contribuições.

As Tabelas 2, 3 e 4 especificam as categorias e as descrições de cada grupo, para que o leitor possa compreender todas as facetas descritas acima e utilizadas neste mapa de estudo. 
Silva, R. F. M.; Burégio, V. A. A.

Revista Gestão.Org, v. 16, Edição Especial, 2018. p. 245-257

ISSN 1679-1827

http://www.revista.ufpe.br/gestaoorg

Tabela 2. Foco da Pesquisa (FACETA 1)

\begin{tabular}{|l|l|} 
Categoria & Descrição \\
\hline Caracterização & $\begin{array}{l}\text { Esta categoria inclui trabalhos focados na caracterização de máquinas sociais, utilizando de um } \\
\text { paradigma, hipótese, teoria, projeto, modelo, framework, linguagem e ferramenta. }\end{array}$ \\
\hline $\begin{array}{l}\text { Plataformas e } \\
\text { Aplicações } \\
\text { Colaborativas }\end{array}$ & $\begin{array}{l}\text { Essa categoria fornece documentos que usam plataformas ou aplicativos de dados colaborativos } \\
\text { para máquinas sociais, como os observatórios da Web, compartilhamento de dados, computação } \\
\text { em nuvem, computação cooperativa, aplicativos de crowdsourcing e cooperação humana. }\end{array}$ \\
\hline $\begin{array}{l}\text { Privacidade e } \\
\text { Dados Pessoais }\end{array}$ & $\begin{array}{l}\text { Esta categoria trabalha com documentos que lidam com a privacidade de dados pessoais em } \\
\text { máquinas sociais, bem como a propriedade de dados, confiança e identidades digitais. }\end{array}$ \\
\hline Arquitetura & Esta categoria tem trabalhos focados na definição de arquiteturas para máquinas sociais. \\
\hline
\end{tabular}

Tabela 3. Tipo de Pesquisa (FACETA 2)

\begin{tabular}{|l|l|}
\hline Categoria & Descrição \\
\hline Pesquisa de Validação & $\begin{array}{l}\text { São investigações de novas técnicas ou metodologias, que ainda não foram } \\
\text { implementadas de forma prática. Os trabalhos são realizados em laboratório, onde são } \\
\text { aplicadas as técnicas ou metodologias da pesquisa. }\end{array}$ \\
\hline Pesquisa de Avaliação & $\begin{array}{l}\text { As técnicas são empregadas em atividades de pesquisa e também são avaliadas por } \\
\text { métodos de estudo. Uma técnica é implementada, e em seguida são verificados os } \\
\text { benefícios, desvantagens e os problemas encontrados. }\end{array}$ \\
\hline Proposta de Solução & $\begin{array}{l}\text { Uma nova proposta ou melhoria de técnica é realizada para resolver um problema. Além } \\
\text { dos benefícios e aplicabilidade da solução, elas podem ser demonstradas com exemplos. }\end{array}$ \\
\hline Artigos Filosóficos & $\begin{array}{l}\text { Esses artigos ilustram novas formas de enxergar problemas existentes, estruturando a } \\
\text { pesquisa por meio de um novo arcabouço conceitual. }\end{array}$ \\
\hline Artigos de Opinião & $\begin{array}{l}\text { Esses artigos mostram como os problemas devem ser resolvidos, ou se a técnica usada } \\
\text { é boa ou ruim, através da opinião pessoal. Esses trabalhos não utilizam trabalhos } \\
\text { relacionados ou pesquisas metodológicas. }\end{array}$ \\
\hline $\begin{array}{l}\text { Estes artigos demonstram a experiência do autor, para explicar como algo foi feito na } \\
\text { prática. }\end{array}$ \\
\hline
\end{tabular}

Tabela 4. Tipo de Contribuição (FACETA 3)

\begin{tabular}{|l|l|}
$\begin{array}{l}\text { Categoria } \\
\begin{array}{l}\text { Procedimento } \\
\text { Guia }\end{array}\end{array}$ & $\begin{array}{l}\text { Descrição } \\
\text { Uma maneira nova ou melhor de fazer uma tarefa específica. Nesta categoria estão incluídas } \\
\text { contribuições como guias, métodos, abordagens ou estratégias. }\end{array}$ \\
\hline $\begin{array}{l}\text { Discussão } \\
\text { Conceitual }\end{array}$ & $\begin{array}{l}\text { Esta categoria trabalha com observações relevantes ou regras práticas, mas não } \\
\text { suficientemente gerais ou sistemáticas para elevar-se ao nível de um modelo descritivo. }\end{array}$ \\
\hline $\begin{array}{l}\text { Modelo Descritivo / } \\
\text { Framework }\end{array}$ & $\begin{array}{l}\text { Um modelo subjetivo, como uma estrutura, taxonomia, ou um framework que aborde uma } \\
\text { determinada área problemática. }\end{array}$ \\
\hline Modelo de Projeto & $\begin{array}{l}\text { Concepção de arquiteturas, modelos de arquitetura para aplicativos e também padrões } \\
\text { arquitetônicos, encaixam nesta categoria como uma forma de colaboração. }\end{array}$ \\
\hline $\begin{array}{l}\text { Algoritmo } \\
\text { Linguagem }\end{array}$ & $\begin{array}{l}\text { Algoritmos, linguagens ou APIs que visam trabalhar no desenvolvimento ou na melhoria de } \\
\text { máquinas sociais. }\end{array}$ \\
\hline Ferramenta & \begin{tabular}{l} 
Tecnologia, sistema ou aplicativo que suportam alguma técnica ou modelo. \\
\hline
\end{tabular}
\end{tabular}


B. Resultados

Com os artigos selecionados e as três facetas de classificação definidas, foi iniciado o meticuloso processo de classificação das obras selecionadas. Este mapa de estudo teve início com a classificação dos trabalhos, utilizando as categorias definidas nas facetas, então em sequência, foi feita uma combinação dos resultados obtidos na etapa anterior.

Na Tabela 5 abaixo, é possível visualizar a classificação da distribuição em cima de todos os trabalhos na faceta do foco da pesquisa, organizada por ano. Uma ressalva extremamente importante deve ser levada em consideração, durante o trabalho de classificação, vários artigos foram classificados em duas ou mais categorias, fazendo a coluna de 2013, por exemplo, ter um somatório maior do que 12 trabalhos classificados, justamente pela classificação dos artigos em mais de uma categoria. O que é naturalmente normal, já que é possível ter um trabalho focado em caracterizar e arquitetar máquinas sociais ao mesmo tempo.

Tabela 5. Distribuição dos estudos por foco de pesquisa por ano

\begin{tabular}{|l|l|l|l|l|l|l|}
\hline & $\mathbf{2 0 1 3}$ & $\mathbf{2 0 1 4}$ & $\mathbf{2 0 1 5}$ & $\mathbf{2 0 1 6}$ & $\mathbf{2 0 1 7}$ & Total \\
\hline Caracterização & 8 & 8 & 11 & 6 & 1 & 34 \\
\hline Plataformas e Aplicações Colaborativas & 5 & 5 & 6 & 4 & 6 & 26 \\
\hline Privacidade e Dados Pessoais & 2 & 2 & 1 & 3 & 3 & 11 \\
\hline Arquitetura & 0 & 1 & 3 & 0 & 2 & 6 \\
\hline
\end{tabular}

Também é possível visualizar na Tabela 5, quais foram as categorias com maior contribuição dos pesquisadores em um determinado ano, observando que de 2013 a 2016, os pesquisadores ainda estavam com um foco muito forte na caracterização das máquinas sociais.

O resultado do mapeamento dentro da faceta foco da pesquisa, resultou em uma distribuição com a seguinte configuração de porcentagem dos trabalhos: $44 \%$ dos trabalhos estão focados em caracterização, 34\% dos trabalhos focados nas plataformas colaborativas e aplicativos, 14\% na privacidade e nos dados pessoais e por fim apenas $8 \%$ com foco em arquitetura.

Os próximos pontos, demonstram onde cada artigo foi classificado, assim o leitor pode visualizar como foram classificados os artigos.

1) Caracterização: como foi mencionado $44 \%$ dos trabalhos estão focados em caracterização de máquinas sociais, uma vez que o tema das máquinas sociais é recente, e com o início das oficinas em 2013 houve uma necessidade significativa de pesquisas nessa área.

Sendo um tema atual, a grande maioria dos pesquisadores tinham o interesse de caracterizar, classificar, conceituar e definir padrões sobre essa questão. Isso é muito mais perceptível, quando se visualiza os tipos das contribuições na coluna de caracterização da Figura 1.

Nesse sentido, há várias propostas que tratam a caracterização de máquinas sociais de forma subjetiva e através de modelos descritivos, assim a comunidade pode iniciar a compreensão das máquinas sociais, os trabalhos que possuem essa visão são observados em (BURÉGIO; MEIRA; ROSA, 2013; CHOPRA; SINGH, 2016; SHADBOLT, et al., 2013; TINAT, et al., 2013; KLEEK, et al., 2015; VASS; MUNSON, 2015; LUCZAK-ROESCH; TINATI; SHADBOLT, 2015; PODDAR, et al., 2016; MATTHEWS, 2016; HALL, 2017; BROWN; HARRIS; HALL, 2015; BURÉGIO, et al., 2013). 
Silva, R. F. M.; Burégio, V. A. A.

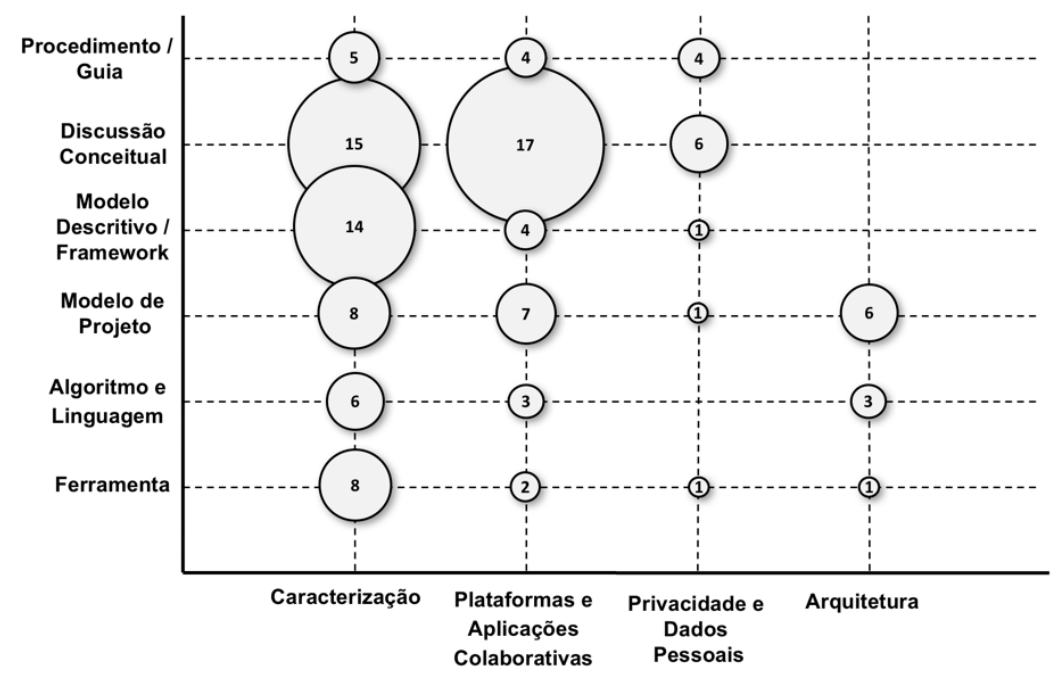

Figura 1. Tipos de contribuições de todos os artigos

Outros trabalhos buscam caracterizar a área por meio de estruturas problemáticas ou modelos subjetivos, utilizando crowdsourcing por exemplo, o conjunto de trabalhos com essas características são vistos em (HALPIN; CAPOCCI, 2014; EVANS et al., 2013; AHLERS, et al., 2016; KLEEK, et al., 2015; STROHMAIER, 2013; DALTON, 2013; MURRAY-RUST, et al., 2015; TARTE, et al., 2015).

Alguns trabalhos desenvolveram pequenos algoritmos, linguagens ou APIs para o entendimento de uma máquina social. Nesse sentido, temos os trabalhos de (NASCIMENTO, et al., 2014; BURÉGIO, et al., 2015; TINATI, et al., 2015; BURÉGIO, et al., 2014; MAAMAR; BURÉGIO; SELLAMI, 2015). Há trabalhos que além do foco inicial, também fornecem um modelo de projeto próprio, ou um software existente para auxiliar na caracterização como em (TINATI, et al., 2013; HALPIN; CAPOCCI, 2014; KLEEK, et al., 2015; LUCZAK-ROESCH; TINATI; SHADBOLT, 2015; SINGH; SHADBOLT, 2013; TARTE; ROURE; WILLCOX, 2014; HALCROW; CARR; HALFORD, 2016; MURRAY-RUST; ROBERTSON, 2014).

Há também um trabalho onde uma máquina social é projetada para uso no contexto de pesquisa inclusiva (HOOPER, et al., 2015). Já no artigo de (MURRAY-RUST, et al., 2014), são apresentados modelos e técnicas para a coordenação de trabalhadores em ambientes multidimensionais de desenvolvimento de software.

Por fim, há alguns trabalhos que caracterizam máquinas sociais utilizando apenas um framework ou uma metodologia, nesse sentido temos os artigos de (GUY; KLEIN, 2014; EVANS et al., 2013; TARTE; ROURE; WILLCOX, 2014; LUCZAK-ROESCH; TINATI; SHADBOLT, 2015).

2) Plataformas e aplicações colaborativas: nesta categoria vários estudos também focaram na caracterização; isso ocorre porque as máquinas sociais têm uma conexão substancial com as plataformas colaborativas, bem como, com os aplicativos de compartilhamento de informações na internet. As pesquisas com máquinas sociais que trabalham de forma colaborativa em plataformas e aplicações, mostraram que apesar de possuírem um percentual significativo, os pesquisadores ainda estão discutindo de forma conceitual o tema discutido e proposto aqui.

Portanto, alguns trabalhos já mencionados também estarão presentes nesta seção, novamente ilustrando como as categorias podem se aglutinar, isso demonstra que os campos de pesquisa não estão concentrados fortemente em apenas um foco ou campo de contribuição.

Os observatórios Web são um meio dedicado ao monitoramento de fatos, eventos e entidades essenciais na rede mundial de computadores, esses observatórios estão presentes nos trabalhos de (ROURE, et al., 2013; KLEEL, et al., 2014; TINATI, et al., 2015; HALCROW; CARR; HALFORD, 2016; MADAAN, et al., 2016; HALL, 2017; BROWN; HARRIS; HALL, 2015). Em outros trabalhos além do observatório, são utilizadas pesquisas com computação colaborativa, cooperação humana ou crowdsourcing, vistos em (KLEEL, et al., 2013; KAJAN, 2017; HARTSWOOD; GRIMPE; JIROTKA, 2013; WANG, et al., 2017; BRAMBILLA, et al., 2014; BRAMBILLA, et al., 2015; TSVETKOVA, et al., 2017; MURRAY-RUST, et al., 2014; GUY; KLEIN, 2014; APPLIN; FISCHER, 2016; HOOPER, et al., 2015; EVANS, et al., 2013; HALL, 2017; ROURE, et al., 2015; GODECKE; ZOLS; 
BACHMAIER, 2013; TINATI; LUCZAK-ROESCH, 2017).

3) Privacidade e Dados Pessoais: algumas das principais preocupações de todas as pessoas que trabalham envolvidas de alguma forma com sistemas, diz respeito à privacidade dos usuários, especialmente quando os dados são compartilhados. Muitos trabalhos discutem a confidencialidade dos dados pessoais e suas propriedades em uso com as máquinas sociais.

Então nessa categoria os artigos presente nessa classificação de privacidade e dados pessoais são esses: (ZHAO, et al., 2016; EVANS, et al., 2013; MERCHANT; JHA; SINGH, 2016; KLEEK, et al., 2013; KLEEK, et al., 2014; KLEEK, et al., 2015; HALL, 2017; TINATI; LUCZAK-ROESCH, 2017; ZHANG, 2017; BINNS; MATTHEWS, 2014). Por fim, o trabalho de HOOPER, et al., (2016) trabalha com dados vinculados, demonstrando como os ecossistemas de máquinas sociais lidam com os problemas de várias partes interessadas, envolvendo questões de motivação, confiança, experiência, segurança, governança, controle, proveniência, privacidade e lei.

4) Arquitetura: o foco principal é a análise e a construção de recursos necessárias para que uma máquina social possa ser construída e desenvolvida, nem sempre se faz obrigatório aprofundar nos detalhes técnicos, mas sim focar nos aspectos que realmente fazem uma arquitetura ser definida, preferencialmente utilizando de padrões arquitetônicos para a construção e definição.

Nesse sentido, foi observado arquiteturas em diversas áreas da computação, logo, há estudos que arquitetam uma descrição da linguagem (NASCIMENTO, et al., 2014), uma arquitetura social orientada para a máquina (BURÉGIO, et al., 2015), uma arquitetura de streaming (TINATI, et al., 2015), uma arquitetura de banco de dados (WANG, et al., 2017), uma arquitetura de armazenamento de dados pessoais (HALL, 2017) e uma arquitetura de dois lados de uma empresa (MAAMAR; BURÉGIO; SELLAMI, 2015).

\section{Discussão}

A. Maturidade das contribuições

O gráfico da Figura 2 abaixo, representa a maturidade das contribuições, nele conclui-se que, o mais extenso conjunto de contribuições sobre máquinas sociais, refere-se a artigos filosóficos e de opiniões, isso demonstra que boa parte da comunidade da engenharia de software bem como alguns autores na área de humanas, estão preocupados em contribuir e compartilhar os conhecimentos adquiridos, para alcançar uma base padrão no campo das máquinas sociais, principalmente para caracterizar, como já dito anteriormente.

Também há uma quantidade significativa de trabalhos que realizam discussões conceituais, isso reforça mais ainda, que as pesquisas na área de máquinas sociais estão em fase de formação e amadurecimento.

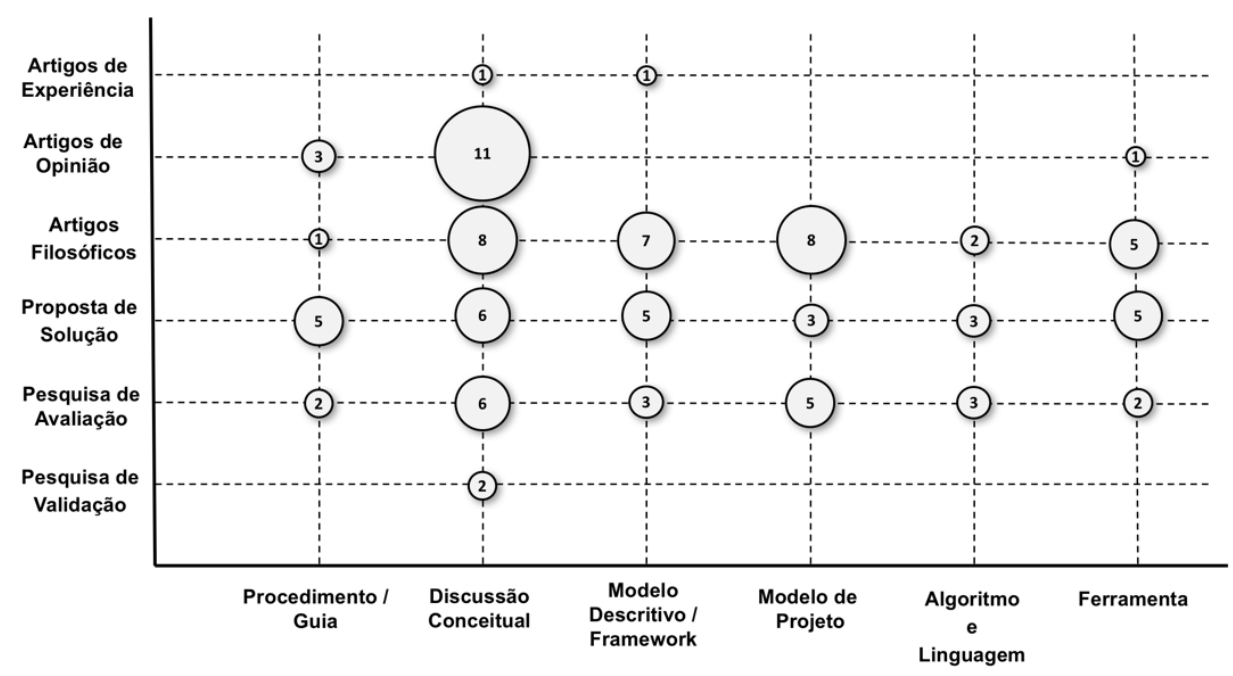

Figura 2. Maturidade das contribuições de todos os artigos

Há poucos estudos que trabalham com privacidade de dados ou com a definição de alguma arquitetura de máquina 
social. Por outro lado, foram identificadas pesquisas em quase todas as dimensões da faceta de tipo de pesquisa, quando cruzadas com a faceta do foco de pesquisa; isso porque os trabalhos analisados pretendem atacar um número significativo de contribuições e de tipos de pesquisas, ou seja, o nível de maturidade da área das máquinas sociais ainda é baixo, pois os pesquisadores ainda não estão em harmonia sobre o assunto ou sobre a direção do problema, atacando assim os problemas em varias direções, o que ocasiona uma grande dispersão dos trabalhos. Por fim, a área de máquina social por ser uma área relativamente recente, há poucos artigos que expressam a experiência do autor ou trabalhos que tratam da evolução de alguma pesquisa existente.

\section{B. Tendências e desafios de pesquisa em máquinas sociais}

Os trabalhos sobre máquinas sociais começaram a aparecer com mais força em 2013, muito se deve ao início das oficinas de máquinas sociais que iniciaram naquele mesmo ano, com isso o campo ainda era pouco explorado, com muitas tentativas de definir um padrão aceito por todas a comunidade, com dúvidas principalmente quanto à segurança e privacidade dos dados pessoais e sem uma arquitetura padronizada para os pesquisadores.

Após um tempo, as pesquisas sobre máquinas sociais direcionaram-se para a observação da internet, na forma de colaboração de dados e também na junção de máquinas sociais com outras áreas emergentes da computação, como internet das coisas ou cidades inteligentes, contudo as pesquisas dispersavam-se cada vez mais de um interesse em comum. Um dos objetivos das máquinas sociais, é a combinação heterogênea de homem e máquina, e com isso resolver problemas para a sociedade, seja na esfera governamental, na saúde ou mesmo no combate à violência.

Assim, com esse mapeamento, foi encontrado um gargalo nas pesquisas que é a definição e construção de algoritmos, $A P I s$, aplicativos ou ferramentas que são configurados como uma máquina social, os estudos nesse sentido ainda são poucos, são recentes, e sem muito aprofundamento. As máquinas sociais têm uma alta tendência de pesquisas, principalmente no campo dos aplicativos, já que homem e máquina estão cada vez mais integrado e interagindo de forma ubíqua, e a sociabilidade atrelada as tecnologias do mundo crescem a cada dia.

Logo, os desafios nessa área são a consolidação e o crescimento das pesquisas em máquinas sociais, após essa consolidação as pesquisas devem focar a atenção em problemas e soluções de maneira mais pontuais no campo das máquinas sociais, por exemplo, a ideia de um "relationship-aware" onde é idealizado que os sistemas devem ser automaticamente conscientes de seus relacionamentos, assim podendo estabelecer uma infinidade de relacionamentos sociais sem conhecer ou saber das propriedades específicas dos outros sistemas.

\section{Ameaças à validade}

É possível destacar algumas ameaças que esse estudo pode apresentar quanto à sua validade: $i$ ) Área de pesquisa recente: as revisões no campo das máquinas sociais são relativamente novas, então os resultados devem ser analisados com cautela, devido ao pequeno número de trabalhos na literatura; ii) Dificuldade nas definições dos campos: ainda não se tem um significado concreto, padronizado e definitivo de todos os campos e pesquisas sobre máquinas sociais, o que pode dificultar a compreensão da área e a definição de tópicos específicos, assim dificultando a criação de novas pesquisas sobre máquinas sociais; iii) Questão de pesquisa: por ter apenas uma pergunta, o leitor pode não encontrar todas as suas respostas; $i v$ ) Obras selecionadas: os artigos mais utilizados foram das oficinas, o que pode tornar os pesquisadores mais focados nos eventos do que na evolução e consolidação da área de máquinas sociais.

\section{Conclusões}

O mapa de estudo mostrou que a maioria dos trabalhos estão focados na caracterização de máquinas sociais, com o objetivo de definir um melhor entendimento da área e também de estabelecer um padrão. Também foi descoberto que existem poucos estudos que implementam algoritmos, APIs, aplicativos ou ferramentas usando as arquiteturas já definidas em máquinas sociais. Se destaca também os tipos de pesquisa mais utilizados pelos autores, a pesquisa de proposta de solução e artigos filosóficos. Então, com base nesse estudo preliminar, há uma relativa falta de pesquisa que aborda o desenvolvimento de uma ferramenta baseada em uma arquitetura, que possa contribuir para a construção de uma máquina social que seja organizada e padronizada, segundo os trâmites da engenharia de software.

Um ponto essencial sobre este trabalho, e que precisa ser mencionado, foi que ele ajudou a detectar as áreas de pesquisa que estão em estudo, os principais tipos de pesquisa, as lacunas existentes e as tendências no campo das máquinas sociais. Além disso, esse mapeamento pode gerar como trabalho futuro, uma revisão sistemática com foco na maturidade das pesquisas da área de máquinas sociais, ou principalmente um trabalho que visa estruturar 
Um Estudo de Mapeamento das Contribuições e Desafios de Pesquisa em Máquinas Sociais Revista Gestão.Org, v. 16, Edição Especial, 2018. p. 245-257

http://www.revista.ufpe.br/gestaoorg

e consolidar o tema, por meio da especificação, projeto e implementação de uma máquina social como referência.

\section{Referências}

AHLERS, D.; DRISCOLL, P.; LÖFSTRÖM, E.; KROGSTIE, J.; WYCKMANS, A. (2016). Understanding Smart Cities as Social Machines. WWW '16 Companion Proceedings of the 25th International Conference Companion on World, p. 759-764, April. ISSN 978-1-4503-4144-8.

APPLIN, S. A.; FISCHER, M. D. (2016). Exploring Cooperation with Social Machines. WWW '16 Companion Proceedings of the 25th International Conference Companion on World Wide Web, p. 765-768, April. ISSN 978-1-4503-4144-8.

BINNS, R.; MATTHEWS, D. (2014). Community structure for efficient information flow in 'ToS; DR', a social machine for parsing legalese. WWW'14 Companion Proceedings of the 23rd International Conference on World Wide Web, p. 881-884, April. ISSN 978-1-4503-2745-9.

BRAMBILLA, M.; CERI, S.; MAURI, A.; VOLONTERIO, R. (2014). Community-based crowdsourcing. WWW '14 Companion Proceedings of the 23rd International Conference on World Wide Web, p. 891-896, April. ISSN 978-1-4503-2745-9.

BRAMBILlA, M.; CERI, S.; MAURI, A; VOLONTERIO, R. (2015). An Explorative Approach for Crowdsourcing Tasks Design. WWW'15 Companion Proceedings of the 24th International Conference on World Wide Web, p. 1125-1130, May. ISSN 978-1-4503-3473-0.

BROWN, I.; HARRIS, L.; HALL, W. (2015). DNA: From Search to Observation Revisited. WebSci '15 Proceedings of the ACM Web Science Conference, p. Article No. 34, June-July. ISSN 978-1-4503-36727.

BURÉGIO, V. A.; MEIRA, S. L.; ROSA, N. S.; GARCIA, V. C. (2013). Moving towards "Relationship-Aware" Applications and Services: A Social Machine-Oriented Approach. 17th IEEE International Enterprise Distributed Object Computing Conference Workshops, p. 43-52, September. ISSN 978-1-4799-3048-7.

BURÉGIO, V. A.; MEIRA, S. L.; ALMEIDA, E. S. D. (2010). Characterizing Dynamic Software Product Lines A Preliminary Mapping Study. Software Product Lines - 14th International Conference, SPLC 2010, p. 5360, September. ISSN 978-1-86220-274-0.

BURÉGIO, V.; NASCIMENTO, L.; ROSA, N.; MEIRA, S. (2014). Personal APIs as an enabler for designing and implementing people as social machines. WWW'14 Companion Proceedings of the 23rd International Conference on World Wide Web, p. 867-872, April. ISSN 978-1-4503-2745-9.

BURÉGIO, V.; BRITO, K.; ROSA, N.; NETO, M.; GARCIA, V.; MEIRA, S. (2015). Towards Government as a Social Machine. WWW'15 Companion Proceedings of the 24th International Conference on World Wide Web, p. 1131-1136, May. ISSN 978-1-4503-3473-0.

BURÉGIO, V.; MEIRA, S.; ROSA, N. (2013). Social Machines: A Unified Paradigm to Describe, Design and Implement Emerging Social Systems. WWW '13 Companion Proceedings of the 22nd International Conference on World Wide Web, p. 885-889, May. ISSN 978-1-4503-2038-2.

CHOPRA, A. K.; SINGH, M. P. (2016). From Social Machines to Social Protocols: Software Engineering Foundations for Sociotechnical Systems. WWW'16 Proceedings of the 25th International Conference on World Wide Web, p. 903-914, April. ISSN 978-1-4503-4143-1.

DALTON, B. (2013). Pseudonymity in social machines. WWW'13 Companion Proceedings of the 22nd International Conference on World Wide Web, p. 897-900, May. ISSN 978-1-4503-2038-2.

EVANS, M. B.; O'HARA, K.; TIROPANIS, T.; WEBBER, C. (2013). Crime applications and social machines: crowdsourcing sensitive data. WWW'13 Companion Proceedings of the 22nd International Conference on World Wide Web, p. 891-896, May. ISSN 978-1-4503-2038-2.

GBAFFONOU, B. A. D.; LAPALME, J.; CHAMPAGNE, R. (2015). Service-Oriented Architecture: A Mapping Study. International Conference on Enterprise Systems (ES), p. 33-42, October. ISSN 978-1-4673-80058 .

GODECKE, A.; ZOLS, W.; BACHMAIER, G. (2013). Ratchet-type micro-hydraulic actuator to mimic muscle 
Silva, R. F. M.; Burégio, V. A. A.

Revista Gestão.Org, v. 16, Edição Especial, 2018. p. 245-257

ISSN 1679-1827

http://www.revista.ufpe.br/gestaoorg

behavior. IECON 2013 - 39 $9^{\text {th }}$ Annual Conference of the IEEE Industrial Electronics Society, p. 39763981, November. ISSN 978-1-4799-0224-8.

GUY, A.; KLEIN, E. (2014). Constructed identity and social machines: a case study in creative media production. WWW ' 14 Companion Proceedings of the $23^{\text {rd }}$ International Conference on World Wide Web, p. 897902, April. ISSN 978-1-4503-2745-9.

HALCROW, C. A.; CARR, L.; HALFORD, S. (2016). Using the SPENCE Model of Online/Offline Community to Analyse Sociality of Social Machines. WWW '16 Companion Proceedings of the $25^{\text {th }}$ International Conference Companion on World Wide Web, p. 769-774, April. ISSN 978-1-4503-4144-8.

HALL, W. (2017). Observing the decentralised social web (one telescope at a time). Workshop on Web Observatories, April.

HALPIN, H.; CAPOCCI, A. (2014). The Berners-Lee hypothesis: power laws and group structure in flickr. WWW ' 14 Companion Proceedings of the $23^{\text {rd }}$ International Conference on World Wide Web, p. 885-890, April. ISSN 978-1-4503-2745-9.

HARTSWOOD, M.; GRIMPE, B.; JIROTKA, M. (2013). Towards Ethical Governance of Social Machines. International Conference on Cloud and Green Computing, p. 426-427, September-October. ISSN 978-07695-5114-2.

HASSAN, M. M.; AFZAL, W.; LINDSTRÖM, B.; SHAH, S. M. A.; ANDLER, S. F.; BLOM, M. (2016). Testability and software performance: a systematic mapping study. SAC '16 Proceedings of the 31st Annual ACM Symposium on Applied Computing, p. 1566-1569, April. ISSN 978-1-4503-3739-7.

HOOPER, C. J.; NIND, M.; PARSONS, S.; POWER, A.; COLLIS, A. (2015). Building a Social Machine: Codesigning a TimeBank for Inclusive Research. WebSci '15 Proceedings of the ACM Web Science Conference, p. Article No. 16, June-July 2015. ISSN 978-1-4503-3672-7.

HOOPER, C. J.; BAILEY, B.; GLASER, H.; HENDLER, J. (2016). Social machines in practice: solutions, stakeholders and scopes. WebSci '16 Proceedings of the $8^{\text {th }}$ ACM Conference on Web Science, p. 156-160, May 2016. ISSN 978-1-4503-4208-7.

KAJAN, E. (2017). Revisiting Interoperability Issues and Challenges in the Era of Ubiquitous Social Web of Everything. ICCES '17 Proceedings of the International Conference on Computing for Engineering and Sciences, p. 1-5, July 2017. ISSN 978-1-4503-5309-0.

KLEEK, M. V.; SMITH, D. A.; HALL, W.; SHADBOLT, N. (2013). "the crowd keeps me in shape": social psychology and the present and future of health social machines. WWW '13 Companion Proceedings of the 22 ${ }^{\text {nd }}$ International Conference on World Wide Web, p. 927-932, May 2013. ISSN 978-1-4503-20382.

KLEEK, M. V.; SMITH, D. A.; TINATI, R.; O'HARA, K.; HALL, W.; SHADBOLT, N. R. (2014). 7 billion home telescopes: observing social machines through personal data stores. WWW '14 Companion Proceedings of the 23 $^{\text {rd }}$ International Conference on World Wide Web, p. 915-920, April 2014. ISSN 978-1-4503-27459 .

KLEEK, M. V.; SMITH, D. A.; MURRAY-RUST, D.; GUY, A.; O'HARA, K.; DRAGAN, L.; SHADBOLT, N. R. (2015). Social Personal Data Stores: the Nuclei of Decentralised Social Machines. WWW '15 Companion Proceedings of the $\mathbf{2 4}^{\text {th }}$ International Conference on World Wide Web, p. 1155-1160, May 2015. ISSN 978-1-4503-3473-0.

LUCZAK-ROESCH, M.; TINATI; SHADBOLT, N. (2015). When Resources Collide: Towards a Theory of Coincidence in Information Spaces. WWW 15 Companion Proceedings of the $24^{\text {th }}$ International Conference on World Wide Web, p. 1137-1142, May 2015. ISSN 978-1-4503-3473-0.

MAAMAR, Z.; BURÉGIO, V.; SELLAMI, M. (2015). Collaborative Enterprise Applications Based on Business and Social Artifacts. IEEE $24^{\text {th }}$ International Conference on Enabling Technologies: Infrastructure for Collaborative Enterprises, p. 150-155, June 2015. ISSN 978-1-4673-7692-1.

MADAAN, A.; TIROPANIS, T.; SRINIVASA, S.; HALL, W. (2016). Observlets: Empowering Analytical Observations on Web Observatory. WWW '16 Companion Proceedings of the $25^{\text {th }}$ International Conference Companion on World Wide Web, p. 775-780, April 2016. ISSN 978-1-4503-4144-8. 
Um Estudo de Mapeamento das Contribuições e Desafios de Pesquisa em Máquinas Sociais Revista Gestão.Org, v. 16, Edição Especial, 2018. p. 245-257

http://www.revista.ufpe.br/gestaoorg

MATTHEWS, P. (2016). Going Meta: Norm Formation and Enactment on the Stack Exchange Network. WWW ' 16 Companion Proceedings of the $25^{\text {th }}$ International Conference Companion on World Wide Web, $p$. 781-785, April 2016. ISSN 978-1-4503-4144-8.

MELDRUM, S.; LICORISH, S. A.; SAVARIMUTHU, B. T. R. (2017). Crowdsourced Knowledge on Stack Overflow: A Systematic Mapping Study. EASE'17 Proceedings of the $21^{\text {st }}$ International Conference on Evaluation and Assessment in Software Engineering, p. 180-185, June 2017. ISSN 978-1-4503-4804-1.

MERCHANT, A.; JHA, T.; SINGH, N. (2016). The Use of Trust in Social Machines. WWW '16 Companion Proceedings of the $2^{\text {th }}$ International Conference Companion on World Wide Web, p. 787-792, April 2016. ISSN 978-1-4503-4144-8.

MURRAY-RUST, D.; SCEKIC, O.; TRUONG, H; ROBERTSON, D.; DUSTDAR, S. (2014). A collaboration model for community-based Software Development with social machines. $\mathbf{1 0}^{\text {th }}$ IEEE International Conference on Collaborative Computing: Networking, Applications and Worksharing, p. 84-93, October 2014. ISSN 978-1-63190-043-3.

MURRAY-RUST, D.; TARTE, S.; HARTSWOOD, M.; GREEN, O. (2015). On Wayfaring in Social Machines. WWW ' 15 Companion Proceedings of the $24^{\text {th }}$ International Conference on World Wide Web, p. 11431148, May 2015. ISSN 978-1-4503-3473-0.

MURRAY-RUST, D.; ROBERTSON, D. (2014). LSCitter: building social machines by augmenting existing social networks with interaction models. WWW ' 14 Companion Proceedings of the $\mathbf{2 3}^{\text {rd }}$ International Conference on World Wide Web, p. 875-880, April 2014. ISSN 978-1-4503-2745-9.

NASCIMENTO, L. M. D.; BURÉGIO, V. A. A.; GARCIA, V. C.; MEIRA, S. R. L. (2014). A new architecture description language for social machines. WWW '14 Companion Proceedings of the $23^{\text {rd }}$ International Conference on World Wide Web, p. 873-874, April 2014. ISSN 978-1-4503-2745-9.

PODDAR, S.; ERNALA, S. K.; SINGH, N.; SAMVARA, A. (2016). Towards a Ubiquitous Model of an Individual in Social Machines. WWW '16 Companion Proceedings of the $\mathbf{2 5}^{\text {th }}$ International Conference Companion on World Wide Web, p. 793-798, April 2016. ISSN 978-1-4503-4144-8.

ROURE, D. D.; HOOPER, C.; MEREDITH-LOBAY, M.; PAGE, K.; TARTE, S.; CRUICKSHANK, D.; ROURE, C. D. (2013). Observing Social Machines Part 1: What to Observe? WWW '13 Companion Proceedings of the $2^{\text {nd }}$ International Conference on World Wide Web, p. 901-904, May 2013. ISSN 978-1-4503-20382 .

ROURE, D. D.; HOOPER, C.; PAGE, K.; TARTE, S.; WILLCOX, P. (2015). Observing Social Machines Part 2: How to Observe? WebSci '15 Proceedings of the ACM Web Science Conference, p. Article No. 13, JuneJuly 2015. ISSN 978-1-4503-3672-7.

SANTOS, A. R.; OLIVEIRA, R. P. D.; ALMEIDA, E. S. D. (2015). Strategies for consistency checking on software product lines: a mapping study. EASE '15 Proceedings of the $\mathbf{1 9}^{\text {th }}$ International Conference on Evaluation and Assessment in Software Engineering, p. Article No. 5, April 2015. ISSN 978-1-4503-33504.

SHADBOLT, N. R.; SMITH, D. A.; SIMPERL, E.; KLEEK, M. V.; YANG, Y.; HALL, W. (2013). Towards a Classification Framework for Social Machines. WWW '13 Companion Proceedings of the $\mathbf{2 2}^{\text {nd }}$ International Conference on World Wide Web, p. 905-912, May 2013. ISSN 978-1-4503-2038-2.

SINGH, P.; SHADBOLT, N. (2013). Linked data in crowdsourcing purposive social network. WWW '13 Companion Proceedings of the $\mathbf{2 2}^{\text {nd }}$ International Conference on World Wide Web, p. 913-918, May 2013. ISSN 978-1-4503-2038-2.

STROHMAIER, M. (2013). A few thoughts on engineering social machines: extended abstract. WWW '13 Companion Proceedings of the $\mathbf{2 2}^{\text {nd }}$ International Conference on World Wide Web, p. 919-920, May 2013. ISSN 978-1-4503-2038-2.

TARTE, S.; WILLCOX, P.; GLASER, H.; ROURE, D. D. (2015). Archetypal Narratives in Social Machines: Approaching Sociality through Prosopography. WebSci '15 Proceedings of the ACM Web Science Conference, p. Article No. 24, June-July 2015. ISSN 978-1-4503-3672-7.

TARTE, S. M.; ROURE, D. D.; WILLCOX, P. (2014). Working out the plot: the role of stories in social machines. WWW '14 Companion Proceedings of the $\mathbf{2 3}^{\text {rd }}$ International Conference on World Wide Web, p. 909- 
914, April 2014. ISSN 978-1-4503-2745-9.

TINATI, R.; CARR, L.; HALFORD, S.; POPE, C. J. (2013). The HTP Model: Understanding the Development of Social Machines. WWW '13 Companion Proceedings of the $\mathbf{2 2}^{\text {nd }}$ International Conference on World Wide Web, p. 921-926, May 2013. ISSN 978-1-4503-2038-2.

TINATI, R.; WANG, X.; BROWN, I.; TIROPANIS, T.; HALL, W. (2015). A Streaming Real-Time Web Observatory Architecture for Monitoring the Health of Social Machines. WWW '15 Companion Proceedings of the $\mathbf{2 4}^{\text {th }}$ International Conference on World Wide Web, p. 1149-1154, May 2015. ISSN 978-1-4503-3473-0.

TINATI, R.; LUCZAK-ROESCH, M. (2017). Wikipedia: a complex social machine by Ramine Tinati and Markus Luczak-Roesch with Martin Vesely as coordinator. ACM SIGWEB Newsletter, p. Article No. 6, February 2017. ISSN 1931-1745.

TINATI, R.; MADAAN, A.; HALL, W. (2017). The Role of Crowdsourcing in the Emerging Internet-Of-Things. WWW '17 Companion Proceedings of the $2^{\text {th }}$ International Conference on World Wide Web Companion, p. 1669-1672, April 2017. ISSN 978-1-4503-4914-7.

TIROPANIS, T.; ROWLAND-CAMPBELL, A.; HALL, W. (2014). Government as a social machine in an ecosystem. WWW '14 Companion Proceedings of the $\mathbf{2 3}^{\text {rd }}$ International Conference on World Wide Web, p. 903-904, April 2014. ISSN 978-1-4503-2745-9.

TSVETKOVA, M.; YASSERI, T.; MEYER, E. T.; PICKERING, J. B.; ENGEN, V.; WALLAND, P.; LÜDERS, M.; FØLSTAD, A.; BRAVOS, G. (2017). Understanding Human-Machine Networks: A Cross-Disciplinary Survey. ACM Computing Surveys (CSUR), p. Article No. 12, April 2017. ISSN 0360-0300.

VASS, J.; MUNSON, J. E. (2015). Revisiting the Three Rs of Social Machines: Reflexivity, Recognition and Responsivity. WWW ' 15 Companion Proceedings of the $24^{\text {th }}$ International Conference on World Wide Web, p. 1161-1166, May 2015. ISSN 978-1-4503-3473-0.

WANG, X.; MADAAN, A.; SIOW, E.; TIROPANIS, T. (2017). Sharing Databases on the Web with Porter Proxy. WWW ' 17 Companion Proceedings of the $26^{\text {th }}$ International Conference on World Wide Web Companion, p. 1673-1676, April 2017. ISSN 978-1-4503-4914-7.

ZHANG, L.; TIROPANIS, T.; HALL, W.; MYAENG, S. (2014). Introducing the omega-machine. WWW '14 Companion Proceedings of the $\mathbf{2 3}^{\text {rd }}$ International Conference on World Wide Web, p. 905-908, April 2014. ISSN 978-1-4503-2745-9.

ZHANG, R. (2017). Alienated Digital Identities. WWW '17 Companion Proceedings of the $\mathbf{2 6}^{\text {th }}$ International Conference on World Wide Web Companion, p. 1677-1678, April 2017. ISSN 978-1-4503-4914-7. 